\title{
Erratum
}

\section{Erratum: Barrientos et al., “Axonal Degeneration Is Mediated by the Mitochondrial Permeability Transition Pore"}

In the article "Axonal Degeneration Is Mediated by the Mitochondrial Permeability Transition Pore," by Sebastian A. Barrientos, Nicolas W. Martinez, Soonmoon Yoo, Juan S. Jara, Sebastian Zamorano, Claudio Hetz, Jeffery L. Twiss, Jaime Alvarez, and Felipe A. Court, which appeared on pages 966-978 of the January 19, 2011 issue, there were errors in two panels in Figure 1G; one of the Western blot lanes was spliced from the original, but the loading control was not spliced accordingly. Dotted lines to show that the original film was spliced have been added to the revised Figure 1 below. In Supplementary Figure 1, an image shown in 2d WT was inadvertently used for $3 \mathrm{~d} \mathrm{Wld}^{s}$; as well, an image in $0 \mathrm{~d} \mathrm{Wld}^{s}$ was shown in the panel corresponding to $1 \mathrm{~d}$ Wld $\mathrm{d}^{\mathrm{s}}$. Images for $1 \mathrm{~d}$ and $3 \mathrm{~d}$ of panel $B$ have been corrected using original raw images for each genotype and condition in Supplementary Figure 1 below. The authors regret this oversight.

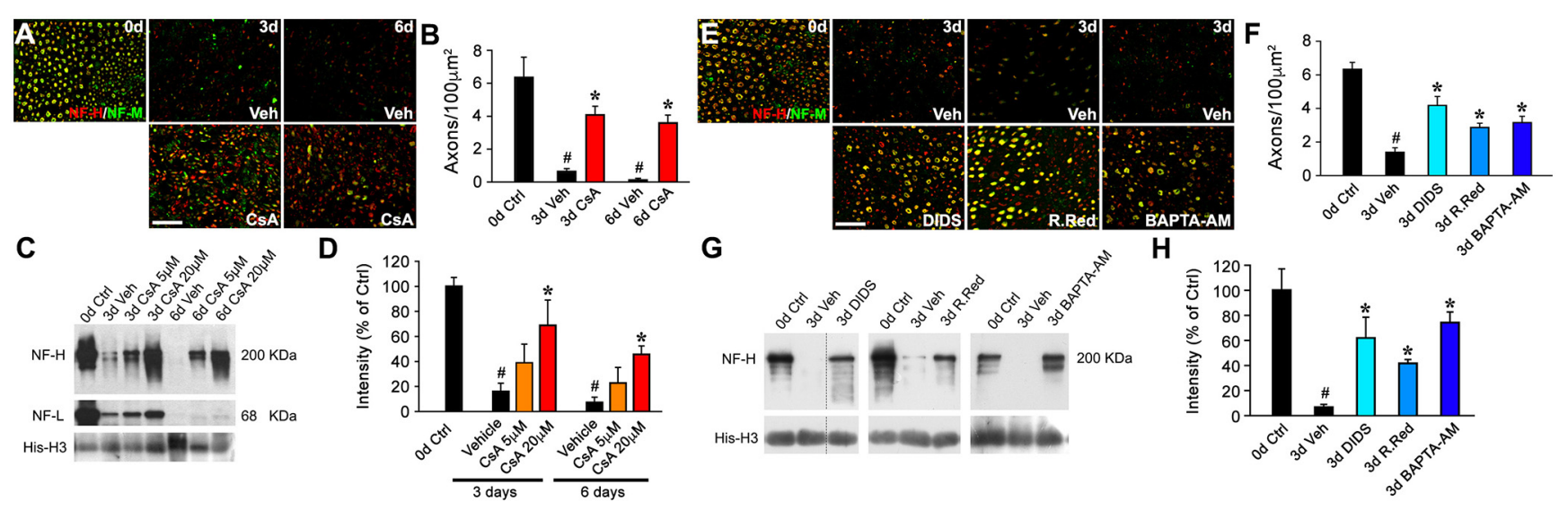

Figure 1. Pharmacological inhibition of mPTP delays axonal degeneration. $A$, Transverse sections of nerve explants stained for NF-H (red) and NF-M (green) isoforms. The NF signals decrease considerably after $3 \mathrm{~d}(3 \mathrm{~d})$ in vehicle solution (Veh) and are almost completely lost by $6 \mathrm{~d}(6 \mathrm{~d})$. The $(s \mathrm{~A}(20 \mu \mathrm{m})$-treated explants show preservation of axonal proteins over 3 and $6 \mathrm{~d}$ in culture. Scale bar, $20 \mu \mathrm{m}$. B, Quantification of NF-H-positive axons in explant cross sections as shown in $\boldsymbol{A}$, expressed as axons per $100 \mu \mathrm{m}^{2}$. Statistically significant protection is seen after CsA treatment at both 3 and $6 \mathrm{~d}(n=3$ per each group; $\# p<0.05$ by Student's $t$ test compared with $0 \mathrm{~d} ; * p<0.05$ by Student's $t$ test compared with $3 \mathrm{~d}$ vehicle; error bars indicate SEM). $C$, Nerve explants after different incubation times and conditions were analyzed by Western blot. Treatment with CsA delays the decrease of NF-H at 3 and $6 \mathrm{~d}$ compared with untreated nerves. The same effect is seen for neurofilament light (NF-L) at $3 \mathrm{~d}$, but less overall protection was seen for NF-L in the 6 d incubation samples. Histone H3 (His-H3) was used as a loading control. $\boldsymbol{D}$, Densitometry of NF-H normalized to His-H3 and expressed as percentage of NF-H at day 0 . Significant protection at $20 \mu \mathrm{m}$ CsA for neurofilament decay is seen at $3 \mathrm{~d}$ and also at $6 \mathrm{~d}$ ( $n=3$ per each group; ${ }^{\#} p<0.05$ by Student's $t$ test compared with $0 \mathrm{~d} ; * p<0.05$ by Student's $t$ test compared with $3 \mathrm{~d}$ vehicle; error bars indicate SEM). $E$, Transverse sections of nerve explants stained for NF-H (red) and NF-M (green). Incubation with the mPTP blockers DIDS $(250 \mu \mathrm{m})$, R.Red $(50 \mu \mathrm{m})$, and BAPTA-AM $(100 \mu \mathrm{m})$ for $3 \mathrm{~d}$ delays axonal degeneration. Scale bar, $20 \mu \mathrm{m}$. $\boldsymbol{F}$, Quantification of axons positive for NF-H in explant cross sections, expressed as axons per $100 \mu \mathrm{m}^{2}$ ( $n=3$ per group; ${ }^{*} p<0.05$ by Student's $t$ test compared with $0 \mathrm{~d}$; $* p<0.05$ by Student's $t$ test compared with $3 \mathrm{~d}$ vehicle; error bars indicate SEM). G, By Western blot analyses for NF-H, each compound appeared to delay the decay of intact NF-H in the nerve explants over $3 \mathrm{~d}$ incubation. $\boldsymbol{H}$, Densitometry of NF-H normalized to His-H3 and expressed as a percentage of NF-H at day 0 . Significant protection of NF decay is seen at $3 \mathrm{~d}(n=3$ per group; ${ }^{\#} p<0.05$ by Student's $t$ test compared with $0 \mathrm{~d} ; * p<0.05$ by Student's $t$ test compared with $3 \mathrm{~d}$ vehicle; error bars indicate SEM).

DOI: $10.1523 /$ JNEUROSCI.2028-21.2021 

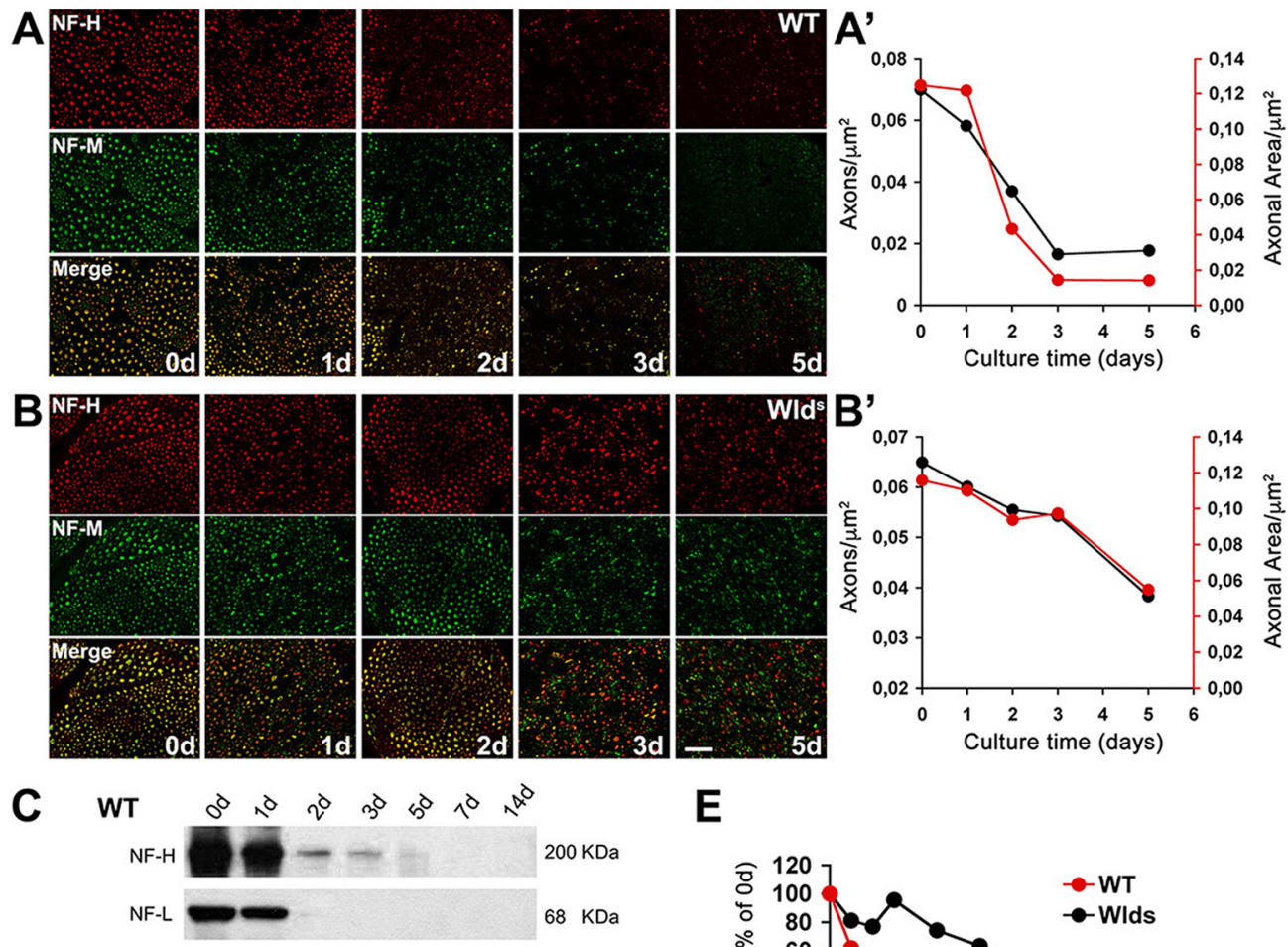

E
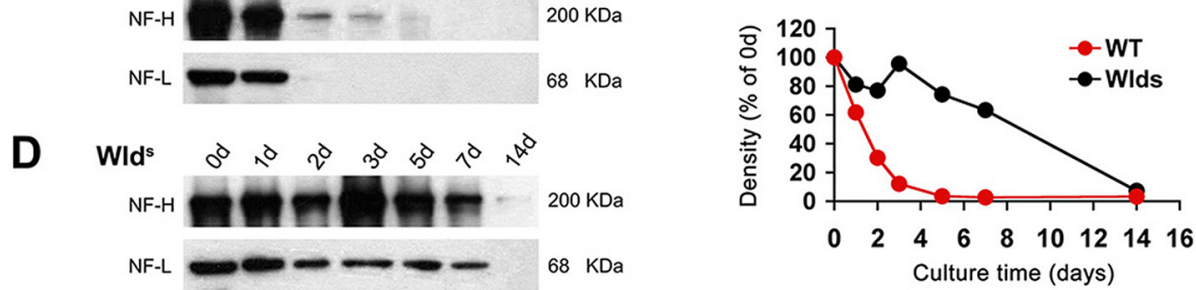

Figure S1. Wallerian degeneration ex vivo. Sciatic nerves from WT (wild-type) and WId ${ }^{5}$ mice were placed in culture dishes and axonal degeneration assessed using quantitative immunofluorescence and Western blot analyses. $\boldsymbol{A}, \boldsymbol{B}$, WT $(\boldsymbol{A})$ and WId ${ }^{5}(\boldsymbol{B})$ sciatic nerve cross sections immunostained for neurofilament isoforms; red, NF-H heavy chain (top row); green, NF-M medium chain (middle row); merged signals (bottom row). The incubation times for each column of images are indicated in the bottom row. $\boldsymbol{A}^{\prime}, \boldsymbol{B}^{\prime}$, Quantification of axon density and area are shown correspondingly in the graph [black symbols and ordinates, axonal density (number of axons per endoneurium area); red symbols and ordinates, endoneurial area occupied by axons (aggregate axonal area per area of nerve)]. $\boldsymbol{A}-\boldsymbol{B}^{\prime}$, The population of WT neurofilament positive axons falls dramatically within the first $3 \mathrm{~d}$ in culture $\left(\boldsymbol{A}, \boldsymbol{A}^{\prime}\right)$, while WId ${ }^{5}$ nerves show clear preservation of axons $\left(\boldsymbol{B}, \boldsymbol{B}^{\prime}\right)$, which is a phenotypic feature of this strain. Scale bar, $20 \mu \mathrm{m} . \boldsymbol{C}, \boldsymbol{D}$, WT $(\boldsymbol{C})$ and WId $(\boldsymbol{D})$ Western blots of neurofilament heavy and light chain expression (NF-H and NF-L, respectively). NF chains fall to very low levels within $3 \mathrm{~d}$ in WT nerves $(\boldsymbol{C})$, whereas in WId ${ }^{5}$ nerves, NF chains remain high for over a week (D). $\boldsymbol{E}$, Quantification of NF-H band. NF-H falls to traces in $3 \mathrm{~d}$ in WT nerves (red curve), while in Wld $\mathrm{s}^{\mathrm{s}}$ nerves (black curve) it remains high for over a week. In a given strain, immunofluorescence or Western blot yields a similar time course of degeneration. 